\title{
On certain $p$-valently analytic functions involving hadamard products
}

\section{Maslina Darus*}

School of Mathematical Sciences, Faculty of Science and Technology, University Kebangsaan Malaysia, Bangi 43600 Selangor, Malaysia

${ }^{\star}$ To whom correspondence should be addressed. E-mail: maslina@pkrisc.cc.ukm.my

Received 12 November 2005

http://dx.doi.org/10.11113/mjfas.v1n1.11

\section{ABSTRACT}

In this paper, we introduce and study some properties of unified class $W_{T}^{\lambda}(\Phi, \Psi ; \eta, \beta, p)$ involving the Hadamard Products given by Juneja et. al in 1985. The properties include coefficient bounds, growth and distortion, and closure theorem. Further, the radii of starlikeness and convexity is also given.

AMS Subj. Classification: $30 \mathrm{C} 45$.

| Convex | Starlike | $p$-valently analytic functions | Hadamard product |

\section{Introduction and Preliminaries}

Denote by $A(p)$ the class of functions of the form

$$
f(z)=z+\sum_{k=1}^{\infty} a_{p+k} z^{p+k}
$$

which are analytic in the open disc $U=\{z: z \in C$ and $|z|<1\}$. Some properties of some subclasses of $A(p)$ were studied by Aouf et. al [11]. Denote by $S^{*}(p, \alpha)$ the class of starlike functions $f \in A(p)$ of order $\alpha(0 \leq \alpha<p)$ satisfying

$$
\operatorname{Re}\left\{\frac{z f^{\prime}(z)}{f(z)}\right\}>\alpha, \quad z \in U
$$


and let $C(p, \alpha)$ be the class of convex functions $f \in A(p)$ of order $\alpha(0 \leq \alpha<p)$ such that $z f^{\prime} \in S^{*}(p, \alpha)$. A function $f \in A(1)$ is said to be in the class of $\beta$-uniformly convex functions of order $\alpha$, denoted by $\beta-U C V(\alpha)[4,5]$ if

$$
\operatorname{Re}\left\{1+\frac{z f^{\prime \prime}(z)}{f^{\prime}(z)}-\alpha\right\} \geq \beta\left|\frac{z f^{\prime \prime}(z)}{f^{\prime}(z)}-1\right|,
$$

and is said to be in a corresponding subclass of $\beta-U C V(\alpha)$, denoted by $\beta-S_{p}(\alpha)$ if

$$
\left\{\frac{z f^{\prime}(z)}{f(z)}-\alpha\right\} \geq \beta\left|\frac{z f^{\prime}(z)}{f(z)}-1\right|
$$

where $-1 \leq \alpha \leq 1$ and $z \in U$.

The class of uniformly convex and uniformly starlike functions has been extensively studied by Goodman[1,2], Ma and Minda[3]. In fact the class of uniformly $\beta$-starlike functions was introduced by Kanas and Wisniowski[9], and for which it can be generalised to $\beta-S_{p}(\alpha)$, the class of uniformly $\beta$-starlike functions of order $\alpha$.

If $f$ of the form (1.1) and $g(z)=z^{p}+\sum_{k=1}^{\infty} b_{p+k} z^{p+k}$ are two functions in $A(p)$, then the Hadamard Product (or Convolution) of $f$ and $g$ is denoted by $f^{*} g$ and given by

$$
(f * g)(z)=z^{p}+\sum_{k=1}^{\infty} a_{p+k} b_{p+k} z^{p+k}
$$

Ruschweyh [7], using the convolution techniques, introduced and studied an important subclass of $A(1)$, the class of prestarlike function of order $\alpha$, which denoted by $R(\alpha)$. Thus $f \in A(1)$ is said to be prestarlike functions of order $\alpha(0 \leq \alpha<1)$ if $f^{*} S_{\alpha} \in S^{*}(\alpha)$ where $S_{\alpha}(z)=\frac{z}{(1-z)^{2(1-\alpha)}}=z+\sum_{n=2}^{\infty} c_{n}(\alpha) z^{n}$ and $c_{n}(\alpha)=\frac{\prod_{j=2}^{n}(j-2 \alpha)}{(n-1) !} \quad(n \in N\{1\} N:=\{1,2,3, \ldots\})$. We note that $R(0)=C(0)$ and $R\left(\frac{1}{2}\right)=S^{*}\left(\frac{1}{2}\right)$. Juneja et. al $[8]$ define the family $D(\Phi, \Psi ; \alpha)$ consisting of function of $f \in A$ so that

$$
\operatorname{Re}\left(\frac{f(z) * \Phi(z)}{f(z) * \Psi(z)}\right)>\alpha, \quad z \in U
$$

where $\Phi(z)=z+\sum_{n=2}^{\infty} \Upsilon_{n} z^{n}$ and $\Psi(z)=z+\sum_{n=2}^{\infty} \gamma_{n} z^{n}$ analytic in $U$ such that $f(z) * \Psi(z) \neq 0, \Upsilon_{n} \geq 0$ and $\Upsilon_{n} \geq \gamma_{n}$ $(n \geq 2)$. We let $D(\Phi, \Psi ; \eta, \beta, p)[10]$ denote the set of all functions in $A(p)$ for which 


$$
\operatorname{Re}\left(1+\frac{1}{\eta}\left(\frac{f(z)^{*} \Phi(z)}{f(z)^{*} \Psi(z)}-p\right)\right)>\beta\left|\frac{1}{\eta}\left(\frac{f(z) * \Phi(z)}{f(z) * \Psi(z)}-p\right)\right|
$$

where $\eta$ is positive real number and $\beta \geq 0$.

For suitable choices of $\Phi, \Psi$, and having $\eta=p-\alpha$, we easily obtain the various subclasses of $A(p)$. For example

$$
\begin{aligned}
& D\left(\frac{z}{(1-z)^{2}}, \frac{z}{1-z} ; p-\alpha, 0\right)=S^{*}(p, \alpha), D\left(\frac{z+z^{2}}{(1-z)^{3}}, \frac{z}{(1-z)^{2}} ; p-\alpha, 0\right)=C(p, \alpha), \\
& D\left(\frac{z+(1-2 \alpha) z^{2}}{(1-z)^{3-2 \alpha}}, \frac{z}{(1-z)^{2-2 \alpha}} ; p-\alpha, 0\right)=R(p, \alpha), D\left(\frac{z}{(1-z)^{2}}, \frac{z}{1-z} ; p-\alpha, \beta\right)=\beta-S_{p}(p, \alpha), \\
& \text { and } \\
& D\left(\frac{z+z^{2}}{(1-z)^{3}}, \frac{z}{(1-z)^{2}} ; p-\alpha, \beta\right)=\beta-U C V(p, \alpha) .
\end{aligned}
$$

Furthermore, note that when $p=1$ we obtain $D\left(\frac{z}{(1-z)^{2}}, \frac{z}{1-z} ; 1-\alpha, 0\right)=S^{*}(\alpha)$,

$$
\begin{aligned}
& D\left(\frac{z+z^{2}}{(1-z)^{3}}, \frac{z}{(1-z)^{2}} ; 1-\alpha, 0\right)=C(\alpha), D\left(\frac{z+(1-2 \alpha) z^{2}}{(1-z)^{3-2 \alpha}}, \frac{z}{(1-z)^{2-2 \alpha}} ; 1-\alpha, 0\right)=R(\alpha), \\
& D\left(\frac{z}{(1-z)^{2}}, \frac{z}{1-z} ; 1-\alpha, \beta\right)=\beta-S_{p}(\alpha), \text { and } D\left(\frac{z+z^{2}}{(1-z)^{3}}, \frac{z}{(1-z)^{2}} ; 1-\alpha, \beta\right)=\beta-U C V(\alpha) .
\end{aligned}
$$

Also denote by $T(p)[6]$ the subclass of $A(p)$ consisting of functions of the form

$$
f(z)=z^{p}-\sum_{k=1}^{\infty} a_{p+k} z^{p+k} .
$$

Now let us write $D_{T}(\Phi, \Psi ; \eta, \beta, p)=D(\Phi, \Psi ; \eta, \beta, p) \cap T(p)$, i.e the class of functions consisting of negative coefficients. All the results obtained concerning the functions of negative coefficients.

\section{Coefficient Inequality}

In this paper we will study the properties of unified presentation of functions $f \in T(p)$ belongs to $W_{T}^{\lambda}(\Phi, \Psi ; \eta, \beta, p)$, i.e a unification of subclass of multivalent starlike and subclass of multivalent convex kind of functions. First of all, we state the following result for the purpose of the study. 
Lemma 2.1 [10] A function $f$ defined by (1.6) is in the class $D_{T}(\Phi, \Psi ; \eta, \beta, p)$ if and only if

$$
\sum_{k=1}^{\infty} \frac{[1+\beta] \Psi_{p+k}-(p(1+\beta)-\eta) \gamma_{p+k}}{\eta-(1+\beta)(p-1)}\left|a_{p+k}\right| \leq 1
$$

where $\eta$ is positive real number, $\beta \geq 0, \Psi_{p+k} \geq 0, \gamma_{p+k} \geq 0$ and $\Psi_{p+k} \geq \gamma_{p+k}$.

Next by observing that

$$
f \in \varepsilon_{T}(\Phi, \Psi ; \eta, \beta, p) \Leftrightarrow z f^{\prime} \in D_{T}(\Phi, \Psi ; \eta, \beta, p)
$$

we obtain the following lemma.

Lemma 2.2 A function $f$ defined by (1.6) is in the class $\varepsilon_{T}(\Phi, \Psi ; \eta, \beta, p)$ if and only if

$$
\sum_{k=1}^{\infty} \frac{(p+k)\left[(1+\beta) \Psi_{p+k}-(p(1+\beta)-\eta) \gamma_{p+k}\right]}{\eta-(1+\beta)(p-1)}\left|a_{p+k}\right| \leq p
$$

where $\eta$ is positive real number, $\beta \geq 0, \Psi_{p+k} \geq 0, \gamma_{p+k} \geq 0$ and $\Psi_{p+k} \geq \gamma_{p+k}$.

In view of Lemma 2.1 and Lemma 2.2, the unification of the class $D_{T}(\Phi, \Psi ; \eta, \beta, p)$ and $\varepsilon_{T}(\Phi, \Psi ; \eta, \beta, p)$ arises naturally and so a new class $W_{T}^{\lambda}(\Phi, \Psi ; \eta, \beta, p)$ is introduced. Thus we say that a function $f$ defined by (1.6) belongs to $W_{T}^{\lambda}(\Phi, \Psi ; \eta, \beta, p)$ if and only if

$$
\sum_{k=1}^{\infty} \frac{\left(1-\lambda+\lambda\left(\frac{p+k}{p}\right)\right)\left[(1+\beta) \Psi_{p+k}-(p(1+\beta)-\eta) \gamma_{p+k}\right]}{\eta-(1+\beta)(p-1)}\left|a_{p+k}\right| \leq 1
$$

Clearly, we obtain

so that

$$
W_{T}^{\lambda}(\Phi, \Psi ; \eta, \beta, p)=(1-\lambda) D_{T}(\Phi, \Psi ; \eta, \beta, p)+\lambda \varepsilon_{T}(\Phi, \Psi ; \eta, \beta, p)
$$

$$
W_{T}^{0}(\Phi, \Psi ; \eta, \beta, p)=D_{T}(\Phi, \Psi ; \eta, \beta, p)
$$

and

$$
W_{T}^{1}(\Phi, \Psi ; \eta, \beta, p)=\varepsilon_{T}(\Phi, \Psi ; \eta, \beta, p)
$$

Next we consider the growth and distortion theorem for the class $W_{T}^{\lambda}(\Phi, \Psi ; \eta, \beta, p)$. We shall omit the proof as the techniques are tedious and standard.

Theorem 2.1 Let the function $f$ defined by (1.6) $W_{T}^{\lambda}(\Phi, \Psi ; \eta, \beta, p)$. Then

$$
\begin{aligned}
& r^{p}-r^{p+1} \frac{\eta-(1+\beta)(p-1)}{\left(1-\lambda+\lambda\left(\frac{p+1}{p}\right)\right)\left[(1+\beta) \Psi_{p+1}-(p(1+\beta)-\eta) \gamma_{p+1}\right]} \leq|f(z)| \\
& r^{p}+r^{p+1} \frac{\eta-(1+\beta)(p-1)}{\left(1-\lambda+\lambda\left(\frac{p+1}{p}\right)\right)\left[(1+\beta) \Psi_{p+1}-(p(1+\beta)-\eta) \gamma_{p+1}\right]}, \quad(|z|=r)
\end{aligned}
$$


and

$$
\begin{aligned}
& p r^{p-1}-r^{p} \frac{(p+1)(\eta-(1+\beta)(p-1))}{\left(1-\lambda+\lambda\left(\frac{p+1}{p}\right)\right)\left[(1+\beta) \Psi_{p+1}-(p(1+\beta)-\eta) \gamma_{p+1}\right]} \leq\left|f^{\prime}(z)\right| \\
& p r^{p-1}+r^{p} \frac{(p+1)(\eta-(1+\beta)(p-1))}{\left(1-\lambda+\lambda\left(\frac{p+1}{p}\right)\right)\left[(1+\beta) \Psi_{p+1}-(p(1+\beta)-\eta) \gamma_{p+1}\right]}, \quad(|z|=r) .
\end{aligned}
$$

The bounds in (2.11) and (2.12) are attained for functions given by

$$
f(z)=z^{p}-z^{p+k} \frac{\eta-(1+\beta)(p-1)}{\left(1-\lambda+\lambda\left(\frac{p+k}{p}\right)\right)\left[(1+\beta) \Psi_{p+k}-(p(1+\beta)-\eta) \gamma_{p+k}\right]}, \quad(k \geq 1) .
$$

Theorem 2.2 Let the function $f$ defined by (1.6) and

$$
g(z)=z^{p}-\sum_{k=1}^{\infty} b_{p+k} z^{p+k}
$$

be in the class $W_{T}^{\lambda}(\Phi, \Psi ; \eta, \beta, p)$. Then the function $f$ defined by

$$
h(z)=(1-\chi) f(z)+\chi g(z)=z^{p}-\sum_{k=1}^{\infty} q_{p+k} z^{p+k}
$$

where $q_{p+k}=(1-\chi) a_{p+k}+\chi b_{p+k}, 0 \leq \chi \leq 1$ is also in the class $W_{T}^{\lambda}(\Phi, \Psi ; \eta, \beta, \lambda, p)$.

Proof. The result follows easily from (2.10) and (2.15).

We prove the following theorem by defining functions $f_{j}(z)(j=1,2,3, \ldots m)$ of the form

$$
f_{j}(z)=z^{p}-\sum_{k=1}^{\infty} a_{p+k, j} z^{p+k} \text { for } \quad a_{p+k, j} \geq 0, \quad z \in U
$$

Theorem 2.3. (Closure theorem) Let the functions $f_{j}(z)(j=1,2,3, \ldots m)$ defined by (2.16) be in the classes $W_{T}^{\lambda}\left(\Phi, \Psi ; \eta_{j}, \beta, p\right) \quad(j=1,2,3, \ldots m)$ respectively. Then the function $h(z)=z^{p}-\frac{1}{m} \sum_{k=1}^{\infty}\left(\sum_{j=1}^{\infty} a_{p+k, j}\right) z^{p+k}$ is the class $W_{T}^{\lambda}(\Phi, \Psi ; \eta, \beta, p)$ where

$$
\eta=\min _{1 \leq j \leq m}\left\{\eta_{j}\right\} \quad \text { with } \quad 0 \leq \eta_{j} \leq 1
$$

Proof. Since $f_{j} \in W_{T}^{\lambda}\left(\Phi, \Psi ; \eta_{j}, \beta, p\right) \quad(j=2,3, \ldots m)$ by applying $(2.10)$, we observe that

$$
\sum_{k=1}^{\infty}\left(1-\lambda+\lambda\left(\frac{p+k}{p}\right)\right)\left[(1+\beta) \Psi_{p+k}-(p(1+\beta)-\eta) \gamma_{p+k}\right]\left(\frac{1}{m} \sum_{j=1}^{\infty} a_{p+k, j}\right)
$$




$$
\begin{aligned}
& =\frac{1}{m} \sum_{j=1}^{\infty}\left(\sum_{k=1}^{\infty}\left(1-\lambda+\lambda\left(\frac{p+k}{p}\right)\right)\left[(1+\beta) \Psi_{p+k}-(p(1+\beta)-\eta) \gamma_{p+k}\right] a_{p+k, j}\right) \\
& \leq \frac{1}{m} \sum_{j=1}^{\infty}\left(\eta_{j}-(1+\beta)(p-1)\right) \leq \eta-(1+\beta)(p-1),
\end{aligned}
$$

which in view of (2.10), again implies that $h \in W_{T}^{\lambda}(\Phi, \Psi ; \eta, \beta, p)$ and the proof is complete.

\section{Radii of Starlikeness and Convexity of $f \in W_{T}^{\lambda}(\Phi, \Psi ; \eta, \beta, p)$}

We first provide the radii of starlikeness for functions $f \in W_{T}^{\lambda}(\Phi, \Psi ; \eta, \beta, p)$.

Theorem 3.4 Let $f \in W_{T}^{\lambda}(\Phi, \Psi ; \eta, \beta, p)$. Then $f$ is starlike of $0 \leq \tau<p$ in $|z|<R_{1}$ where

$$
R_{1}=\inf _{k \geq 1}\left[\frac{(p-\tau)\left(1-\lambda+\lambda\left(\frac{p+k}{p}\right)\right)\left[(1+\beta) \Psi_{p+k}-(p(1+\beta)-\eta) \gamma_{p+k}\right]}{(p+k-\tau)[\eta-(1+\beta)(p-1)]}\right]^{\frac{1}{p+k-1}} .
$$

Proof. It is sufficient to prove

$$
\left|\frac{z f^{\prime}(z)}{f(z)}-p\right|<p-\tau \text {. }
$$

For the left hand side of (3.18) we have

$$
\begin{aligned}
& \left|\frac{z f^{\prime}(z)}{f(z)}-p\right|=\left|\frac{\sum_{k=1}^{\infty}(-k) a_{p+k} z^{p+k-1}}{1-\sum_{k=1}^{\infty} a_{p+k} z^{p+k-1}}\right| \\
& \leq \frac{\sum_{k=1}^{\infty} k a_{p+k}|z|^{p+k-1}}{1-\sum_{k=1}^{\infty} a_{p+k}|z|^{p+k-1}} .
\end{aligned}
$$

This last expression is less than $(p-\tau)$ since

$$
|z|^{p+k-1}<\frac{(p-\tau)\left(1-\lambda+\lambda\left(\frac{p+k}{p}\right)\right)\left[(1+\beta) \Psi_{p+k}-(p(1+\beta)-\eta) \gamma_{p+k}\right]}{(p+k-\tau)[\eta-(1+\beta)(p-1)]} .
$$

Therefore the proof is complete.

Using the fact that $f$ is convex if and only if $z f^{\prime}$ is starlike, we obtain the following: 
Theorem 3.5 Let $f \in W_{T}^{\lambda}(\Phi, \Psi ; \eta, \beta, p)$. Then $f$ is convex order $0 \leq \tau<p$ in $|z|<R_{2}$ where

$$
R_{2}=\inf _{k \geq 1}\left[\frac{(p-\tau)\left(1-\lambda+\lambda\left(\frac{p+k}{p}\right)\right)\left[(1+\beta) \Psi_{p+k}-(p(1+\beta)-\eta) \gamma_{p+k}\right]}{(p+k)(p+k-\tau)[\eta-(1+\beta)(p-1)]}\right]^{\frac{1}{p+k-1}}
$$

We omit the proof as it is easily derived.

Finally, we obtain our last result stated in the following theorem.

Theorem 3.6 Let $f \in W_{T}^{\lambda}(\Phi, \Psi ; \eta, \beta, p)$. Then $f$ is close-to-convex order $0 \leq \tau<p$ in $|z|<R_{3}$ where

$$
R_{3}=\inf _{k \geq 1}\left[\frac{(p-\tau)\left(1-\lambda+\lambda\left(\frac{p+k}{p}\right)\right)\left[(1+\beta) \Psi_{p+k}-(p(1+\beta)-\eta) \gamma_{p+k}\right]}{(p+k)[\eta-(1+\beta)(p-1)]}\right]^{\frac{1}{p+k-1}}
$$

Again we omit the proof as it is easily derived.

\section{Acknowledgement}

The work present here is supported by IRPA grant 09-02-02-10029 EAR, Malaysia.

\section{References}

[1] W. Goodman, Ann. Polon. Math., 56 (1991) 87-92.

[2] W. Goodman, J. Math. Anal. \& Appl., 155 (1991), 364-370

[3] W. Ma and D. Minda, Ann. Polon. Math., 57 (1992) 165-175.

[4] F. Rønning, Proc. Amer. Math. Soc., 118 (1993) 189-196.

[5] F. Rønning, Ann. Polon. Math., 60 (1995), 289-297.

[6] H. Silverman, Proc. Amer. Math. Soc., 51 (1975) 109-116.

[7] S. Ruschweyh, Comm. Math. Helv., 52 (1977) 497-509.

[8] Juneja, T. R. Reddy and M. L. Mogra, Soochow J. Math., 11 (1985), 69-81.

[9] S. Kanas and A. Wisniowska, Rev. Roumanie Math. Pures Appl. 45 (2000) 647-657.

[10] M. Darus, accepted in JIPAM.

[11] M. K. Aouf, M. Hossen and H. M. Srivastava, Appl. Math. Comp., 39 (2000) 39-48. 